\title{
Intraspecific trait variability and community assembly in hawkmoths (Lepidoptera: Sphingidae) across an elevational gradient in the eastern Himalayas, India
}

\author{
Mansi Mungee * \\ ORCID ID - 0000-0002-5452-7196 \\ mansim@students.iiserpune.ac.in \\ Indian Institute of Science Education and Research \\ Homi Bhabha Road, Pashan, Pune - 411008, Maharashtra, INDIA.
}

\section{Ramana Athreya}

ORCID ID - 0000-0001-7141-7311

rathreya@iiserpune.ac.in

Indian Institute of Science Education and Research

Homi Bhabha Road, Pashan, Pune - 411008, Maharashtra, INDIA.

\section{* Corresponding author}

Funding: This work was partly funded by the Department of Science and Technology, Government of India (Grant No. SR/SO/AS66/2011) and by the Nadathur Trust, Bengaluru. PhD scholarship for MM was funded by Indian Institute of Science Education and Research, Pune (IISER Pune).

Author contributions: MM conceived this particular investigation into traits, performed the analysis and drafted the manuscript. RA is the PI of the larger moth diversity project, 
and supervised the analysis and manuscript writing. All the rest, including collection and curation of field and trait data, were shared by both.

Acknowledgments: The authors would like to thank Deepak Barua for stimulating our interest in functional ecology and for the several rounds of discussions that led to the synthesis of this paper. We thank the Forest Department of Arunachal Pradesh for their assistance and research permits (CWL/G/13(95)/2011-12/Pt.II/660-62 during 2011-2015). This work would not have been possible without the enormous support of diverse kinds provided by Mr. Indi Glow, Nima Tsering and other members of the Singchung Bugun community.

Conflicts of interest: None. 


\section{Abstract}

1. Recent progress in functional ecology has advanced our understanding of the role of intraspecific (ITV) and interspecific (STV) trait variation in community assembly across environmental gradients. Studies on plant communities have generally found STV as the main driver of community trait variation, whereas ITV plays an important role in determining species co-existence and community assembly. However, similar studies of faunal taxa, especially invertebrates, are very few in number.

2. We investigated variation of hawkmoth (Lepidoptera: Sphingidae) traits along an environmental gradient spanning $2600 \mathrm{~m}$ in the eastern Himalayas and its role in community assembly, using the morpho-functional traits of body mass (BM), wing loading (WL) and wing aspect ratio (AR).

3. We employ the recently proposed T-statistics to test for non-random assembly of hawkmoth communities and the relative importance of the two opposing forces for trait divergence (internal filters) and convergence (external filters).

4. Community-wide trait-overlap decreased for all three traits with increasing environmental distance, suggesting the presence of elevation specific optimum morphology (i.e. functional response traits). Community weighted mean of BM and AR increased with elevation. Overall, the variation was dominated by species turnover but ITV accounted for $25 \%, 23 \%$ and $<1 \%$ variability of $B M, W L$ and $A R$, respectively. T-statistics, which incorporates ITV, revealed that elevational communities had a non-random trait distribution, and that community assembly was dominated by internal filtering throughout the gradient.

5. This study was carried out using easily measurable morpho-traits obtained from calibrated field images of a large number (3301) of individuals. That these also happened to be important environmental response traits resulted in a significant signal in the metrics that we investigated. Such studies of abundant and 
hyperdiverse invertebrate groups across large environmental gradients should considerably improve our understanding of community assembly processes.

31 Key words: community assembly, intraspecific variance, T-statistics, Sphingidae,

32 invertebrates 


\section{1. Introduction}

34 Convergence, and divergence of traits of co-occurring species highlight the effects of two

35 ecological processes that govern community assembly. The abiotic environment, via

36 environmental filtering, causes trait convergence by imposing constraints on the range of

37 trait values, regardless of species, that facilitate their persistence in a habitat (Weiher et

38 al., 1998). On the other hand, competitive interactions are expected to cause trait

39 divergence by limiting the extent of ecological similarity (and hence 'debilitating'

40 competition) 'permissible' for co-occurring species in a community (Macarthur \& Levins,

41 1967). Consequently, community assembly depends on an interplay between both, biotic

42 interactions and environmental filtering, acting over ecological and evolutionary time-

43 scales.

45 Several metrics of 'functional diversity' (FD) proposed in recent years (Villéger et al., 46 2008; Mouchet et al., 2010) allow the assessment of the relative importance of these two 47 ecological processes and contributed to an improved understanding of community 48 assembly mechanisms (Albert et al., 2010; Baraloto et al., 2012; Pigot et al., 2016).

50 However, these metrics, while useful, have ignored several other processes of community

51 assembly such as equalizing fitness or facilitation (Grime, 2006; Butterfield \& Callaway;

52 2013). Additionally, most metrics of FD assume that the relative importance of the two

53 assembly processes is similar across different traits, thereby overlooking the importance of

54 trade-offs in trait filtering (e.g. Spasojevic \& Suding; 2012). FD indices also ignore the 55 effects of intraspecific variability (ITV), i.e. they assume that trait variation among 56 individuals of a species is negligible as compared to variation across species. However, 57 this assumption has rarely been empirically validated (but see Albert et al., 2010; Jung et 58 al., 2010) and almost all such attempts have been for plants (but see Griffiths et al., 2016 
59 for a case study on dung beetles) and similar investigations are still lacking for

60 invertebrates which form the bulk of terrestrial biodiversity (see Brosseau et al., 2018;

61 Wong et al., 2019 for recent reviews).

62

63 A recently proposed suite of functional trait metrics, T-statistics (Violle et al., 2012),

64 incorporate ITV into their calculations. They classified the different processes into the two

65 broad categories of 'external' and 'internal' filters. While the external filters include all

66 assembly processes outside the community that are responsible for 'filtering' species from

67 the regional pool (e.g. environmental constraints, predatory pressures, etc.), the internal

68 filters refer to assembly processes internal to the community, i.e. micro-environmental

69 heterogeneity and density-dependent processes that facilitate coexistence within the

70 community. They used the variance ratios of functional traits across taxonomic (individual,

71 population, species and community) and spatial (local and regional) scales to identify the

72 dominant operational filter.

74 This identification of two ecological filters, provides a clearer, even if somewhat broad,

75 picture of their relative importance in the establishment and persistence of traits and taxa

76 in the community through the comparison of intra- and interspecific trait variation at local

77 and regional scales (Hulshof et al., 2013; Le Bagousse-Pinguet et al., 2014; Luo et al.,

78 2016; Neyret et al., 2016; Outreman et al., 2017; Xavier Jordani et al., 2019).

80 The application of this metric to animal taxa has been constrained by the difficulty in

81 identifying the minimum set of traits needed to adequately describe species' resource axes

82 and in obtaining large scale, individual level trait measurements. Such databases are more

83 easily obtained for plants, which lack behavioral and movement related responses, and 84 where not only the traits, but also their relation to the individuals' fitness, or their 
85 functionality, are easily quantifiable (Lavorel et al., 2013; Lamanna et al., 2014).

87 We present here the distribution patterns of three key morphological traits - body mass

88 (BM), wing loading (WL) and wing aspect ratio (AR) - of 3301 individual hawkmoths

(Lepidoptera: Sphingidae) across a large environmental gradient at multiple taxonomic and spatial scales. We have analysed these patterns to assess (i) the functional role of these traits and (ii) the relative importance of external (e.g. environment) and internal (e.g. competition) filters, vis-a-vis randomness, in structuring elevational communities. The environmental gradient was derived by a linear combination of temperature, precipitation, air density and productivity across $2600 \mathrm{~m}$ of elevation in the eastern Himalayas.

More specifically, we test the following hypotheses -

(i) The degree of overlap of the distribution of traits across communities should be anticorrelated with the environmental distance between them as the three traits have been previously linked to organismal performance (Sinervo \& Huey, 1990; Hammond et al., 2000; Berwaerts et al., 2002; Huyghe et al., 2005; McGill et al., 2006) through thermoregulation and flight (Dudley, 2002; Dillon et al., 2006)

(ii) The community mean trait values of (a) BM should increase with elevation (better thermoregulation associated with larger bodies), (b) WL should decrease and AR should increase with elevation (improved flight efficiency at higher elevations where resources are generally sparse and air density is lower),

(iii) If hawkmoth communities are governed by the same widespread pattern seen in other taxa (Seifert et al., 2015), the change in community mean trait along the environmental gradient will be dominated by species turnover rather than intraspecific variation, and pool and, internal filters should dominate community trait structure. 


\section{2. Methods \& Materials}

\section{Study area and Field Sampling}

113 Hawkmoth sampling was carried out in Eaglenest Wildlife Sanctuary (hereafter EWS), a

114 Protected Area of $218 \mathrm{~km}^{2}$ located between $27^{\circ} 02^{\prime \prime} 09^{\prime} \mathrm{N}$ and $92^{\circ} 18^{\prime \prime} 35^{\prime} \mathrm{E}$ in the eastern

115 Himalayas of Arunachal Pradesh, northeast India, during April - July 2014 (Figure 1). The

116 high diversity of this region, believed to be due to its complex terrain and its location at the

117 confluence of the Oriental and Sino-Japanese floristic and faunistic zones (Holt et al.,

118 2013) makes it a globally important biodiversity hotspot (Orme et al., 2005). The large

119 altitudinal range of $3000 \mathrm{~m}$ coupled with high rainfall $(>3000 \mathrm{~mm}$ along the southern

120 slopes) has resulted in diverse habitat types ranging from tropical wet evergreen below

$121900 \mathrm{~m}$ to coniferous temperate forests above $2800 \mathrm{~m}$ (Choudhury, 2003). The sampling, in

122 the form of point surveys at UV illuminated screens on no-moon nights, was carried out

123 along a vehicle track characterized by roadside scrub in very close proximity to primary

124 forest (5-20 m away). The 12 elevations between $500 \mathrm{~m}$ and $2800 \mathrm{~m}$ were clustered in a

125 small stretch spanning just $15 \mathrm{~km}$. The $200 \mathrm{~m}$ location, near the village of Tippi, was

126 separated from its nearest neighbor by about $20 \mathrm{~km}$ due to the lack of access to suitable

127 habitat along this road (Figure 1).

129 The sampling was limited to a single compact transect to minimize the bias due to a

130 variation in gamma diversity while sampling across distant transects (McCain, 2007). We

131 sampled at 2-5 elevations simultaneously to sample across the elevational gradient with

132 some degree of uniformity of weather conditions (which can change drastically from day

133 to day). Hawkmoth individuals arriving at a light screen were photographed against the

134 reference grid (on the screen) using consumer-grade point-and-shoot digital cameras.

135 Following Willott (2001) our sampling strategy attempted to equalize the number of 136 individuals, rather than trap nights, across elevations to minimize the large diurnal variation 
137 in moth numbers at a light screen, even within the no-moon window. A total of 4731

138 hawkmoth individuals, spanning all 3 Sphingid subfamilies, 30 genera and 80 139 morphospecies, were recorded from across 13 elevations.

\section{Species identification and trait measurement}

142 We assigned individuals to morpho-species using the online resources made available by 143 Kitching and collaborators (e.g. Kitching, 2019). We obtained the primary measurements 144 of body length, thorax width, wing costum length and wing breadth from which we derived 145 the three functional traits of body mass (BM), wing loading (WL) and wing aspect ratio (AR) from field images after calibration and distortion corrections (Mungee \& Athreya, 2019). Traits were reliably measured for 3301 images (69\% individuals) spanning 76 morphospecies and 30 genera making it the first systematic compilation of any insect trait data from the region. Details of the sampling methodology and trait measurement are provided as Supporting information (Supplementary S1).

\section{Environmental variables}

153 We used 4 environmental variables including mean annual temperature (MAT), mean 154 annual precipitation (APPT), productivity (EVI: enhanced vegetation index) and air density (AD). These variables were strongly correlated with each other and all decreased with elevation. A principal component analysis showed that the first two axes explained $91.4 \%$ and $7.7 \%$ of the variance. Therefore, we used PC-1, which has a strong linear relationship 158 with elevation, as a composite environmental variable, hereafter referred to as the 159 Environment in italics (see Supplementary S1 for a detailed analysis).

\section{Diversity and Trait data set}

162 Traits were measured for only a subset (trait data set: 3301 individuals; $69 \%$ ) of the total 
163 number of individuals identified to morphospecies (diversity data set: 4731 individuals).

164 We carried out all analyses using just the trait data set, and also with the diversity data set;

165 in the latter we filled in the missing traits by randomly drawing trait values from other

166 individuals of that species at that elevation. For example, only 66 of the 79 individuals of

167 Acosmerycoides harterti at $700 \mathrm{~m}$ had measured traits. The remaining 13 individuals were

168 assigned trait values drawn from the set of 66 measurements (random sampling with

169 replacement). We did not simulate these extra values using the population mean and

170 standard deviations as this would have affected the statistics of the sample mean and

171 dispersion. The results for both treatments are quite similar. In any case, an assessment of

172 the completeness of our samples using taxonomic rarefaction (for diversity data set) and

173 functional rarefaction (for the trait data set) curves was done (Supplementary S1;

174 Figures 6 \& 7 respectively). Additionally, since the fraction of individuals with traits was 175 very low at $1700 \mathrm{~m}$ (due to poor weather), we carried out the analysis with and without this 176 elevation.

178 The analysis with the trait data set including $1700 \mathrm{~m}$ is shown here; the same analysis with 179 (i) trait data set without $1700 \mathrm{~m}$ and (ii) diversity data set with $1700 \mathrm{~m}$ are shown in 180 Supplementary S2.

\section{Trait variation across the environmental gradient}

183 We used two approaches to examine the functional response of hawkmoth communities 184 across the environmental gradient. First, we investigated the change of functional 'alpha' 185 diversity across the gradient using the community abundance-weighted mean trait value 186 (CWM; Lavorel et al., 2008). Two different CWM values were analysed for each 187 community, CWM1 - using the regional species mean trait value, i.e. mean across all 188 elevations, and CWM2 - using the population mean trait values, i.e. mean across all 
189 individuals of a species at an elevation:

191 where $a_{i k}$ is the relative abundance of species $i$ at elevation $k, t_{i}$ is the regional mean trait

192 value for species $i$, and $t_{i k}$ is the population mean for species $i$ at elevation $k$. The change

193 of CWMs with environment was assessed using the ordinary least squares (OLS) 194 regression.

Second, we quantified the change in trait across the gradient by the degree of overlap of the kernel density distributions for all pairs of communities (Mouillot et al., 2005), i.e essentially the functional 'beta' diversity. The kernel density distributions were constructed in a non-parametric manner and do not assume an underlying distribution for community trait values. The distribution function for each trait at each elevation was calculated as the sum of kernel density functions for each individual in that community. The degree of overlap of a trait between any pair of communities was simply the area of overlap of their

203 trait density distributions. We used OLS regressions to examine change of overlap with 204 environmental distance. We did not estimate the multivariate overlap as either the average 205 overlap across all traits, or the overlap in multidimensional space (Mouillot et al., 2005).

206 The difference in the slopes for different traits, i.e. the rate of response to the same environmental gradient, can be a measure of the strength of their functional response.

\section{Variance decomposition}

210 We partitioned the community-level response of hawkmoth traits to the environmental 211 gradient into species turnover (STV) and intraspecific variation (ITV) following the 212 approach of Lepš et al., (2011). 
215 We used linear discriminant analysis (LDA; Venables \& Ripley, 2002) to assess if the

216 hawkmoth species were well discriminated by the four primary traits (body length, thorax 217 width, wing costum length and wing breadth). This helped to quantify the role of

218 intraspecific trait variation in confounding the assignment of an individual to its species on

219 the basis of (just) these four traits.

\section{T-statistics}

222 We first log-transformed the trait values to remove potential scaling effects between

223 measurements. We calculated three variance ratios (T-statistics, Violle et al., 2012) at 224 nested spatial and taxonomic scales as follows:

- $\mathrm{T}_{\mathrm{IP} / \mathrm{IC}}=\frac{\sigma_{I P}^{2}}{\sigma_{I C}^{2}}$, where $\sigma_{\mathrm{IP}}^{2}$ is the variance of trait values among individuals within a population, and $\sigma^{2}{ }_{\text {Ic }}$ is the variance of trait values among individuals within a community (strength of internal filtering).

- $\mathrm{T}_{\mathrm{ICIR}}=\frac{\sigma_{I C}^{2}}{\sigma_{I R}^{2}}$, where $\sigma^{2}{ }_{\mathrm{IR}}$ is the variance of trait values among individuals within the regional pool (strength of external filtering acting on individuals)

- $\mathrm{T}_{\mathrm{PC} / \mathrm{PR}}=\frac{\sigma_{P C}^{2}}{\sigma_{P R}^{2}}$, where, $\sigma^{2} \mathrm{PC}$ is the variance of population mean trait values within a community and $\sigma^{2}$ PR is the variance of population mean trait values within the regional pool (strength of internal filtering acting on species)

234 The observed metrics were compared to those obtained from the simulated null models to detect non-random assembly of community traits. Details on generation of the null models

236 are provided in Supplementary S3. The standardized effect sizes (SES) were calculated 


$$
S E S=\frac{I_{o b s}-I_{\text {null }}}{\sigma_{\text {null }}}
$$

239 where $I_{\text {obs }}$ is the observed value for a given index, $I_{\text {null }}$ is the mean of the simulated null

240 replicates, and $\sigma_{\text {null }}$ their standard deviation. OLS regressions were used to assess the

241 relationship between individual metrics and the composite environmental variable and also

242 for $\mathrm{T}_{\mathrm{IP} / \mathrm{IC}}$ versus species richness (Violle et al., 2012).

244 All above analyses were performed in the R programming software; version 3.4 .4 (R

245 Development Core Team, 2015) and the following packages were used: vegan 2.5.4 for

246 computing species richness, diversity indices, taxonomic rarefaction curves and

247 environmental variables PCA scores (Oksanen et al., 2019); evolqg 0.2.6 for functional 248 rarefaction curves (Melo et al., 2015); FD 1.0.12 for CWM analysis (Laliberté et al., 2010); 249 sfsmisc 1.1-3 for the trait kernel density analysis (Maechler, 2019); MASS 7.3.51.4 for 250 LDA (Venables \& Ripley, 2002) and corrplot 0.84 for the associated plot (Wei \& Simko, 251 2017); cati 0.99 .2 for calculating the T-statistics and generating null models; function 252 decompCTRE for variance partitioning (Taudiere \& Violle, 2016).

\section{Results}

255 Trait variation across the environmental gradient

256 Community weighted mean of BM exhibited a significant positive relationship with 257 Environment (Figure 2). The slope of CWM2, that incorporates intraspecific variation in it's 258 calculation was slightly greater than CWM1. WL did not exhibit any significant relationship 259 with Environment using either CWM1 or CWM2. CWM of AR showed a significant positive 260 correlation with Environment and similar to BM, the slope of CWM2, was marginally 261 greater than CWM1 (Table 1). We used Fisher's r-to-z transformation to compare the two 262 fits. The difference between the slopes of CWM1 and CWM2 were not significant for any 
265 LDA analysis yielded a mean attrition probability of $63 \%$ for correctly attributing an

266 individual to its species from its trait values (Supplementary S3, Figure 1).

Kernel density plots for each trait-elevation distribution are shown in Supplementary S3;

Figure 2. The reduction of trait overlap with environmental distance (Figure 3) was significant for all traits (Table 1). Slopes were significantly different between BM and AR

271 (Fisher's r-to-z transformations, Supplementary S3; Table 3). Trait overlap did not exhibit

272 any significant pattern with absolute elevation, for any of the three traits (Supplementary

S3; Table 4).

\section{Variance decomposition}

276 The maximum source of variation in all three traits was species turnover $(B M=70 \%, W L=$ $27750 \%$, and $A R=99 \%)$, followed by intraspecific variation $(B M=25 \%, W L=23 \% \& A R=$ $278<1 \%$; Figure 4). This was also reflected in the high species turnover across the entire gradient (Supplementary S3; Figure 3)

\section{T-statistics}

282 The observed metrics of T-statistics and their SES values are listed in Supplementary S3;

283 Tables 5-8. TIP/IC was significantly lower than the null model at all elevations and for all 284 three traits (Figure 5). Distribution of $\mathrm{T}_{I C / R}$ was more variable: BM was significantly lower 285 than null at some of the lowest $(200,500,900 \mathrm{~m})$ and highest $(2500 \& 2700 \mathrm{~m})$ elevations 286 and higher than null in between $(1100,1300,1500$, and $1700 \mathrm{~m})$. SES for $\mathrm{T}_{\text {IC/R }}$ value for 287 WL was significantly lower than null at most elevations, but higher at $200 \mathrm{~m}$. AR was 288 mostly not significantly different from null, except at $200 \mathrm{~m}$ (lower) and $700 \mathrm{~m}$ (higher). $\mathrm{T}_{\mathrm{PC} /}$ PR Was not significantly different from null at any elevation, and for any trait (Figure 5). We 
290 also observed that the correlation of SES values with Environment was significantly

291 negative for $T_{I P / I C}$ of $B M$ and $W L$, and significantly positive for $T_{I C / R}$ of $W L$. $T_{I P / I C}$ was not

292 found to be significantly correlated with rarefied species richness for any of the three traits

293 (Supplementary S3; Figure 4).

\section{Discussion}

This study involves the first systematic collection of individual-level trait data for any invertebrate group from the study region. We are not aware of any previous work that uses trait variance ratios via $T$-statistics to explore community assembly of invertebrate fauna over a continuous gradient and across multiple sites (but see Outreman et al., 2017). This work also highlights the importance of morphological traits as key functional 'responses' when the traits can be directly implicated in individual survival, or performance strategies.

We tested the hypothesis that tropical hawkmoth communities of eastern Himalayas are not randomly assembled with respect to key morphological traits. We demonstrated that body and wing sizes are important functional attributes that respond to a changing environment across a large elevational gradient, and the strength of this response did not change significantly upon incorporation of intraspecific variability. Community mean body mass and aspect ratio increased with Environment (i.e. elevation), however, wing loading

309 did not exhibit any directional variation along the gradient. Using a trait data from 3301 individuals, we showed strong internal filtering in hawkmoth communities, which indicates low niche overlap among co-occurring species. The strength of the external filtering varied

312 with trait and environment. More importantly, external filtering acted on individuals rather 313 than species highlighting the importance of incorporating intraspecific variance in understanding community assembly processes. 


\section{Trait variation across the environmental gradient}

317 Several frameworks and guidelines have been suggested for consideration in trait

318 selection for faunal groups (for most recent reviews see Luck et al. 2012 for vertebrate

319 groups and Brosseau et al., 2018 \& Wong et al., 2019 for invertebrates).

321 Morphological traits are easily quantifiable, and some can be directly linked to individual

322 function and survival, especially when the performance trait has a simple mechanical basis

323 in design (Wainwright, 1988). Especially for hawkmoths, which are a facultatively

324 endothermic group (thermoregulation by active shivering of thoracic muscles; Heinrich

325 1996), body mass can be considered a key response trait determining species' functional

326 strategies along a wide elevation (temperature) gradient. It should be noted that while

327 body mass may be governed by multiple life history traits (e.g. reproductive age,

328 starvation, etc), body volume (our measurements) will have a more predictable relationship

329 with thorax muscle mass. Wing loading and wing aspect ratio are two important

330 determinants of flight performance, and consequently fitness (e.g. reproduction via mate-

331 locating, host-plant searching, and survival via predator escape, resource acquisition and

332 dispersal).

334 A larger body size at low ambient temperatures is consistent with better thermoregulatory 335 properties for facultatively endothermic hawkmoths, sensu lato Bergmann's Pattern 336 (Salewski \& Watt, 2017). Previously reported patterns of variation in body size for 337 invertebrates along temperature gradients have been idiosyncratic to taxon and spatial 338 scale of investigation (Shelomi, 2012; Vinarski, 2014; Brehm et al., 2019). Body size also 339 has direct implications for several important processes such as physiological, macro340 ecological and evolutionary (Blackburn \& Gaston, 1994). Thus further tests of the relative 341 importance of these different causative mechanisms will be required to ascribe the strong 
342 observed pattern to a definite process.

344 The adaptations for powered flight can be broken down into two key wing attributes - (i)

345 ratio of body mass to wing area (wing loading), and (ii) functional variations in wing shape,

346 especially in the length of the wing relative to the width (aspect ratio) (Hassall, 2015). Our

347 results indicate that hawkmoth communities exhibit strong adaptations for better dispersal

348 abilities and flight efficiency via increased aspect ratio, but not wing-loading. In

349 vertebrates, higher aspect ratio (longer, thinner wings) is found to give faster and more

350 efficient flight and has been shown to be associated with migratory species in birds

351 (Vágási et al., 2016). For insects, while there are several theoretical speculations that

352 lower aspect ratio may be more suited at higher air densities (due to higher viscous forces

353 experienced by small objects, and due to the difference in the number, structure and

354 locomotory independence of wings between insects and higher vertebrates), empirical

355 results have been mixed (Hassall, 2015; Bai et al., 2016). A higher aspect ratio for

356 communities of hawkmoths at higher elevations, is indicative of adaptations to long

357 dispersal flights which may be better suited to the patchiness in resource distributions

358 more commonly associated with regions of low productivity, as suggested by the EVI

359 values of high elevations in our data. Interestingly, wing loading did not exhibit a significant

360 trend with elevation indicating different selection pressures on wing shape and size.

362 Trait overlap decreased with increasing Environmental distance between communities

363 confirming the role of the three morphological traits in functional response strategies of the

364 hawkmoth communities along an elevational gradient that exhibits a large range of

365 temperature, productivity and air density. Many studies have demonstrated

366 correspondence between species morphological traits (morphospace) and their

367 'performance' or functional strategies (Price et al., 2014; Pigot et al., 2015; Dehling et al., 
2016). For instance, Pigot et al. (2015) found that key dimensions of the ecological niche in passerines, including diet, foraging maneuver and foraging substrate were, to varying extents, predictable on the basis of morphological traits. As agents of many ecosystem processes and services, the morphological response of non-producers merits further

372 research.

\section{Variance Partitioning}

Species turnover and intraspecific variation accounted for $73 \%$ and $19 \%$, respectively, of the total trait variance. This not insignificant contribution of ITV is consistent with a growing body of literature advocating the use of both individual and species-specific traits to investigate community assembly mechanisms (Jung et al., 2010). As seen in plant communities, the contribution of intraspecific variation was strongly dependent on the trait, with $25 \%$ contribution for BM, and less than $1 \%$ for AR. Very few studies have explored the extent of intraspecific variability in insect communities till date, and obtained very contrasting values (< 5\% for dung beetles, Insecta: Coleoptera, by Griffiths et al., 2016; < 1\% for stonefly assemblages, Insecta: Plecoptera, by Garcia-Raventós et al., 2017; and > 70\% for spiders, Arachnida: Araneae, by Dahirel et al., 2017). Other taxa have reported mixed results (33\% for tadpoles by Xavier Jordani et al., 2019; $70 \%$ in lichens by Asplund \& Wardle, 2014). In general, it is suggested that community-level trait variation, especially across broad environmental gradients, is driven primarily by species turnover, but the 388 relative importance of intraspecific variation depends strongly on the trait, environmental 389 factor, and spatial scale considered. The variation observed across studies for invertebrates warrants further investigations to propose general assembly mechanisms.

392 We note here that while intraspecific variability played a lesser role in the change of the 393 community mean trait value with Environment, the LDA analysis of species-trait robustness 
394 (Supplementary S3, Figure 1) suggests a substantial spread of values away from the

395 species mean.

398 The realized and fundamental niches of co-occurring species, are key to understanding 399 how local communities are assembled from a 'regional' species pool (Kraft et al., 2008). 400 Using T-statistics, we demonstrated the non-random assembly of hawkmoth communities 401 and classify them based on their assembly forces i.e. internal and external filtering. The 402 metric $\mathrm{T}_{\mathrm{IP} / \mathrm{IC}}$, indicative of internal filtering, showed that individuals of a species within a 403 local community were more closely clustered with each other in trait space, than may be 404 expected from a random chance. Similar patterns of non-overlapping distribution of 405 morphological traits among co-occurring species has been reported previously for tadpoles 406 (Xavier Jordani et al., 2019) and for plant communities across environmental gradients 407 (Luo et al., 2016; Neyret et al., 2016). Interestingly, the SES values of TIP/IC exhibited a 408 negative relationship with Environment for body mass and wing loading, suggesting a 409 decrease in the strength of internal filtering with elevation (i.e. increasing environmental 410 severity).

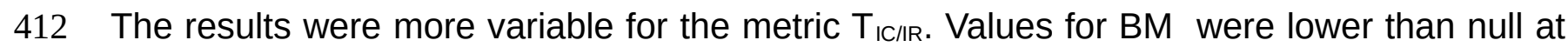
413 some of the highest and lowest elevations, but those for WL were consistently lower than 414 null for most communities (i.e. individuals within a community are more similar to each 415 other than individuals across communities), indicative of external filtering. External 416 pressures, perhaps the stressful environment (low productivity, temperature and air density 417 at high elevations) and anthropogenic or predator pressures (at low elevations), are acting 418 as a strong filter allowing only individuals with specific trait values to persist in these 419 communities. Values higher than the null distribution for certain communities is a 
420 potentially interesting result and indicates that the individuals here exhibit high variance in

421 their trait values, compared to the variance in trait values of individuals across all

422 communities and thus, these communities are assembled with weak external filtering.

423 Importantly, similar patterns of external filtering were not observed at the species level $\left(T_{\mathrm{PC} /}\right.$

$424 \mathrm{PR})$, when only interspecific variation was used. This supports the importance of

425 incorporating intraspecific variation in examination of community assembly patterns (Jung

426 et al., 2010).

\section{CONCLUSION}

429 We carried out a study of intraspecific traits of the hawkmoth community across a $2600 \mathrm{~m}$

430 elevational transect in Eaglenest wildlife sanctuary in north-east India. We obtained a 431 diversity data set of 4731 individuals spanning 80 species and 30 genera, of which we

432 could measure traits for 3301 individuals. This is the first such systematic study of 433 intraspecific variation of any taxon from this globally important biodiversity hotspot. We 434 found that the three traits - body mass, wing loading and wing aspect ratio, which are 435 implicated in thermoregulation and flight - change in response to a changing environment 436 across that elevational gradient. As a community, hawkmoths exhibited larger body sizes, 437 lower wing loading and higher aspect ratio at higher elevations. Species turn-over 438 dominated these changes but the intraspecific variation was not insignificant; furthermore, 439 its contribution changed with the trait. We also used a metric from the suite of T-statistics 440 to infer the role of internal filtering in community assembly. Although changes in 441 community-average trait values across the environmental gradient may be discerned by 442 (regional) species-level means, as has been the dominant strategy of investigations, one 443 can/should expect local community dynamics to be largely influenced by intraspecific trait 444 variation. Indeed, another T-statistic metric showed a significant signal only when 445 individual trait values were used but not with species means. Only plant researchers seem 
446 to have used T-statistics to investigate internal and external filters affecting community

447 assembly, except for two, recent, studies on hymenopterans (Outreman et al., 2017) and

448 tadpoles (Xavier Jordani et al., 2019). Thus, this study is perhaps the first such of a group

449 from the hyperdiverse Lepidoptera. 


\section{References}

453

454

455

456

457

458

459

460

1. Albert, C.H., Thuiller, W., Yoccoz, N.G., Douzet, R., Aubert, S. and Lavorel, S., 2010. A multi-trait approach reveals the structure and the relative importance of intra- vs. interspecific variability in plant traits - Funct. Ecol., 24(6):1192-1201.

2. Asplund, J. and Wardle, D.A., 2014. Within-species variability is the main driver of community-level responses of traits of epiphytes across a long-term chronosequence. - Funct. Ecol., 28(6),1513-1522.

3. Athreya, R., 2006. Eaglenest biodiversity project (2003-2006): conservation resources for Eaglenest wildlife sanctuary. Kaati Trust, Pune.

4. Bai, Y., Dong, J.J., Guan, D.L., Xie, J.Y. and Xu, S.Q., 2016. Geographic variation in wing size and shape of the grasshopper Trilophidia annulata (Orthoptera: Oedipodidae): morphological trait variations follow an ecogeographical rule. Scientific reports, 6, p.32680.

5. Baraloto, C., Hardy, O.J., Paine, C.T., Dexter, K.G., Cruaud, C., Dunning, L.T., Gonzalez, M.A., Molino, J.F., Sabatier, D., Savolainen, V. and Chave, J., 2012. Using functional traits and phylogenetic trees to examine the assembly of tropical tree communities. - J. Ecol., 100(3),690-701.

6. Berwaerts, K., Van Dyck, H. and Aerts, P., 2002. Does flight morphology relate to flight performance? An experimental test with the butterfly Pararge aegeria. Funct. Ecol., 16(4),484-491.

7. Blackburn, T.M. and Gaston, K.J., 1994. Animal body size distributions: patterns, mechanisms and implications. - Trends Ecol. Evol., 9(12),471-474.

8. Brehm, G., Zeuss, D. and Colwell, R.K., 2019. Moth body size increases with elevation along a complete tropical elevational gradient for two hyperdiverse clades. - Ecography, 42(4),632-642.

9. Brousseau, P.M., Gravel, D. and Handa, I.T., 2018. On the development of a predictive functional trait approach for studying terrestrial arthropods. - J. Anim. Ecol., 87(5),1209-1220.

10. Butterfield, B.J. and Callaway, R.M., 2013. A functional comparative approach to facilitation and its context dependence. - Funct. Ecol., 27(4),907-917.

11. Choudhury, Anwaruddin. "Birds of Eaglenest Wildlife Sanctuary and Sessa Orchid Sanctuary, Arunachal Pradesh, India." - Forktail (2003): 1-14.

12. Dahirel, M., Dierick, J., De Cock, M. and Bonte, D., 2017. Intraspecific variation shapes community-level behavioral responses to urbanization in spiders. Ecology, 98(9),2379-2390.

13. Dehling, D.M., Jordano, P., Schaefer, H.M., Böhning-Gaese, K. and Schleuning, M., 2016. Morphology predicts species' functional roles and their degree of specialization in plant-frugivore interactions. - Proc R Soc Lond [Biol], 283(1823), p.20152444. 
14. Dillon, M.E., Frazier, M.R. and Dudley, R., 2006. Into thin air: physiology and evolution of alpine insects. - Integr Comp Biol., 46(1),49-61.

15. Dudley, R., 2002. The biomechanics of insect flight: form, function, evolution. Princeton University Press.

16. Garcia-Raventós, A., Viza, A., Tierno de Figueroa, J.M., Riera, J.L. and Múrria, C., 2017. Seasonality, species richness and poor dispersion mediate intraspecific trait variability in stonefly community responses along an elevational gradient. Freshwater Biol., 62(5),916-928.

17. Griffiths, H.M., Louzada, J., Bardgett, R.D. and Barlow, J., 2016. Assessing the importance of intraspecific variability in dung beetle functional traits. - PLoS One, 11(3), p.e0145598.

18. Grime, J.P., 2006. Trait convergence and trait divergence in herbaceous plant communities: mechanisms and consequences. - J. Veg. Sci., 17(2),255-260.

19. Hammond, K.A., Chappell, M.A., Cardullo, R.A., Lin, R.S. and Johnsen, T.S., 2000. The mechanistic basis of aerobic performance variation in red junglefowl. - J. Exp. Biol., 203(13),2053-2064.

20. Hassall, C., 2015. Strong longitudinal variation in wing aspect ratio of a damselfly, Calopteryx maculata (Odonata: Zygoptera). - PeerJ PrePrints, 3, p.e998v1.

21. Heinrich, B., 1996. The thermal warriors: strategies of insect survival. Harvard University Press.

22. Holt, B.G., Lessard, J.P., Borregaard, M.K., Fritz, S.A., Araújo, M.B., Dimitrov, D., Fabre, P.H., Graham, C.H., Graves, G.R., Jønsson, K.A. and Nogués-Bravo, D., 2013. An update of Wallace's zoogeographic regions of the world. - Science, 339(6115),74-78.

23. Hulsey, C.D. and García de León, F.J., 2005. Cichlid jaw mechanics: linking morphology to feeding specialization. - Funct. Ecol., 19(3),487-494.

24. Hulshof, C.M., Violle, C., Spasojevic, M.J., McGill, B., Damschen, E., Harrison, S. and Enquist, B.J., 2013. Intra-specific and inter-specific variation in specific leaf area reveal the importance of abiotic and biotic drivers of species diversity across elevation and latitude. - J. Veg. Sci., 24(5),921-931.

25. Huyghe, K., Vanhooydonck, B., Scheers, H., Molina $\square$ Borja, M. and Van Damme, R., 2005. Morphology, performance and fighting capacity in male lizards, Gallotia galloti. - Funct. Ecol., 19(5),800-807.

26. Jung, V., Violle, C., Mondy, C., Hoffmann, L. and Muller, S., 2010. Intraspecific variability and trait-based community assembly. - J. Ecol., 98(5),1134-1140.

27. Kitching, I.J. 2019. Sphingidae Taxonomic Inventory, http://sphingidae.myspecies.info/, accessed on 20 May, 2016 
28. Kraft, N.J., Valencia, R. and Ackerly, D.D., 2008. Functional traits and niche-based tree community assembly in an Amazonian forest. - Science, 322(5901),580-582.

29. Laliberté, E., Legendre, P., Shipley, B. and Laliberté, M.E., 2014. Package 'FD'.

34. Lehmann, F.O., 1999. Ambient temperature affects free-flight performance in the fruit fly Drosophila melanogaster. - J. Comp. Physiol., 169(3),165-171.

35. Lepš, J., de Bello, F., Šmilauer, P. and Doležal, J., 2011. Community trait response to environment: disentangling species turnover vs intraspecific trait variability effects. - Ecography, 34(5),856-863.

36. Luck, G.W., Lavorel, S., McIntyre, S. and Lumb, K., 2012. Improving the application of vertebrate trait $\square$ based frameworks to the study of ecosystem services. - J. Ecol., 81(5),1065-1076.

37. Luo, Y., Liu, J., Tan, S., Cadotte, M.W., Xu, K., Gao, L. and Li, D., 2016. Trait variation and functional diversity maintenance of understory herbaceous species coexisting along an elevational gradient in Yulong Mountain, Southwest China. Plant Div., 38(6),303-311.

38. MacArthur, R. and Levins, R., 1967. The limiting similarity, convergence, and divergence of coexisting species. - Am. Nat., 101(921),377-385.

39. Maechler, M., Stahel, W., Ruckstuhl, A., Keller, C., Halvorsen, K., Hauser, A. and Buser, C., 2019. Package 'sfsmisc'.

40. McCain, C.M., 2007. Area and mammalian elevational diversity. - Ecology, 88(1),76-86.

41. McGill, B.J., Enquist, B.J., Weiher, E. and Westoby, M., 2006. Rebuilding community ecology from functional traits. - Trends Ecol. Evol., 21(4),178-185. 
42. Melo, D., Garcia, G., Hubbe, A., Assis, A.P. and Marroig, G., 2015. EvolQG-An R package for evolutionary quantitative genetics. F1000Research, 4.

43. Mouchet, M.A., Villéger, S., Mason, N.W. and Mouillot, D., 2010. Functional diversity measures: an overview of their redundancy and their ability to discriminate community assembly rules - . Funct. Ecol., 24(4),867-876.

44. Mouillot, D., Stubbs, W., Faure, M., Dumay, O., Tomasini, J.A., Wilson, J.B. and Do Chi, T., 2005. Niche overlap estimates based on quantitative functional traits: a new family of non-parametric indices. - Oecologia, 145(3),345-353.

45. Mungee, M. and Athreya, R., 2019. High throughput photogrammetric measurement of morphological traits in free-ranging phototropic insects. - BioRxiv, p.699454.

46. Neyret, M., Bentley, L.P., Oliveras, I., Marimon, B.S., Marimon-Junior, B.H., Almeida de Oliveira, E., Barbosa Passos, F., Castro Ccoscco, R., dos Santos, J., Matias Reis, S. and Morandi, P.S., 2016. Examining variation in the leaf mass per area of dominant species across two contrasting tropical gradients in light of community assembly - . Ecol. Evol., 6(16),5674-5689.

47. Oksanen, J., Kindt, R., Legendre, P., O'Hara, B., Stevens, M.H.H., Oksanen, M.J. and Suggests, M.A.S.S., 2007. The vegan package. Community ecology package, 10,631-637.

48. Orme, C.D.L., Davies, R.G., Burgess, M., Eigenbrod, F., Pickup, N., Olson, V.A., Webster, A.J., Ding, T.S., Rasmussen, P.C., Ridgely, R.S. and Stattersfield, A.J., 2005. Global hotspots of species richness are not congruent with endemism or threat. - Nature, 436(7053), p.1016.

49. Outreman, Y., Andrade, T.O., Louâpre, P., Krespi, L., Violle, C. and Van Baaren, J., 2018. Multi-scale and antagonist selection on life-history traits in parasitoids: $A$ community ecology perspective. Func. Ecol.,32(3), pp.736-751.

50. Pigot, A.L., Trisos, C.H. and Tobias, J.A., 2016. Functional traits reveal the expansion and packing of ecological niche space underlying an elevational diversity gradient in passerine birds. - Proc R Soc Lond [Biol], 283(1822), p.20152013.

51. Price, T.D., Hooper, D.M., Buchanan, C.D., Johansson, U.S., Tietze, D.T., Alström, P., Olsson, U., Ghosh-Harihar, M., Ishtiaq, F., Gupta, S.K. and Martens, J., 2014. Niche filling slows the diversification of Himalayan songbirds. - Nature, 509(7499), p.222.

52. Salewski, V. and Watt, C., 2017. Bergmann's rule: a biophysiological rule examined in birds. - Oikos, 126(2).

53. Shelomi, M., 2012. Where are we now? Bergmann's rule sensu lato in insects. Am. Nat., 180(4),511-519.

54. Sinervo, B. and Huey, R.B., 1990. Allometric engineering: an experimental test of the causes of interpopulational differences in performance. - Science, 248(4959),1106-1109. 
55. Spasojevic, M.J. and Suding, K.N., 2012. Inferring community assembly mechanisms from functional diversity patterns: the importance of multiple assembly processes. - J. Ecol., 100(3),652-661.

56. Taudiere, A. and Violle, C., 2016. cati: an R package using functional traits to detect and quantify multi-level community assembly processes. - Ecography, 39(7),699708.

57. Vágási, C.I., Pap, P.L., Vincze, O., Osváth, G., Erritzøe, J. and Møller, A.P., 2016. Morphological adaptations to migration in birds. - Evol. Biol., 43(1),48-59.

58. Venables, W.N., Ripley, B.D., 2002. Modern Applied Statistics with S. fourth ed. Springer, New York.

59. Villéger, S., Mason, N.W. and Mouillot, D., 2008. New multidimensional functional diversity indices for a multifaceted framework in Funct. Ecol.. - Ecology, 89(8),2290-2301.

60. Vinarski, M.V., 2014. On the applicability of Bergmann's rule to ectotherms: the state of the art. - Biol. Bull. Rev., 4(3),232-242.

61. Violle, C., Enquist, B.J., McGill, B.J., Jiang, L.I.N., Albert, C.H., Hulshof, C., Jung, V. and Messier, J., 2012. The return of the variance: intraspecific variability in community ecology. - Trends Ecol. Evol., 27(4),244-252.

62. Wainwright, P.C., 1988. Morphology and ecology: functional basis of feeding constraints in Caribbean labrid fishes. - Ecology, 69(3),635-645.

63. Wei, T., Simko, V., Levy, M., Xie, Y., Jin, Y. and Zemla, J., 2017. Package 'corrplot'. Statistician, 56,316-324.

64. Weiher, E., Clarke, G.P. and Keddy, P.A., 1998. Community assembly rules, morphological dispersion, and the coexistence of plant species. - Oikos,309-322.

65. Wong, M.K., Guénard, B. and Lewis, O.T., 2019. Trait-based ecology of terrestrial arthropods. - Biol.I Rev., 94(3),999-1022.

66. Wootton, R.J., 1992. Functional morphology of insect wings. - Ann. Rev. Entom., $37(1), 113-140$.

67. Xavier Jordani, M., Mouquet, N., Casatti, L., Menin, M., de Cerqueira Rossa-Feres, D. and Albert, C.H., 2019. Intraspecific and interspecific trait variability in tadpole meta-communities from the Brazilian Atlantic rainforest. - Ecol. Evol., 9(7),40254037. 


\section{Table 1. Variation of hawkmoth community traits with Environment}

639 Results from the linear regression analysis involving community traits and the Environment

640 (i.e. composite environmental variable, which is also strongly correlated with elevation).

641 The traits used are body mass (BM), wing loading (WL) and wing aspect ratio (AR). The

642 analysis was carried out for both population mean trait values weighted by local

643 abundance (CWM1) and regional species mean trait values weighted by local abundance

644 (CWM2).

\begin{tabular}{|c|c|c|c|c|}
\hline \multicolumn{5}{|c|}{ Community mean trait values with Environment } \\
\hline Trait & Intercept \pm SE & Slope \pm SE & Adj. $\mathbf{R}^{2}$ & p.value \\
\hline BM (CWM1) & $1.98 \pm 0.03$ & $0.05 \pm 0.02$ & 0.39 & $<0.05^{\star}$ \\
\hline BM (CWM2) & $1.98 \pm 0.04$ & $0.06 \pm 0.02$ & 0.34 & $<0.05^{\star}$ \\
\hline WL (CWM1) & $(5.88 \pm 0.07) \times 10^{-3}$ & $(-5.90 \pm 4.02) \times 10^{-5}$ & 0.09 & 0.17 \\
\hline WL (CWM2) & $(5.86 \pm 0.11) \times 10^{-3}$ & $(-8.05 \pm 5.77) \times 10^{-5}$ & 0.07 & 0.19 \\
\hline AR (CWM1) & $3.54 \pm 0.01$ & $0.02 \pm 0.01$ & 0.55 & $<0.005^{\star \star}$ \\
\hline AR (CWM2) & $3.54 \pm 0.01$ & $0.03 \pm 0.01$ & 0.61 & $<0.005^{\star \star}$ \\
\hline \multicolumn{5}{|c|}{ Trait overlap with Environmental distance } \\
\hline BM & $0.88 \pm 0.01$ & $-0.03 \pm 0.01$ & 0.29 & $<0.005^{\star \star}$ \\
\hline WL & $0.89 \pm 0.02$ & $-0.03 \pm 0.01$ & 0.19 & $<0.05^{\star}$ \\
\hline AR & $0.90 \pm 0.01$ & $-0.02 \pm 0.004$ & 0.25 & $<0.005^{\star \star}$ \\
\hline
\end{tabular}



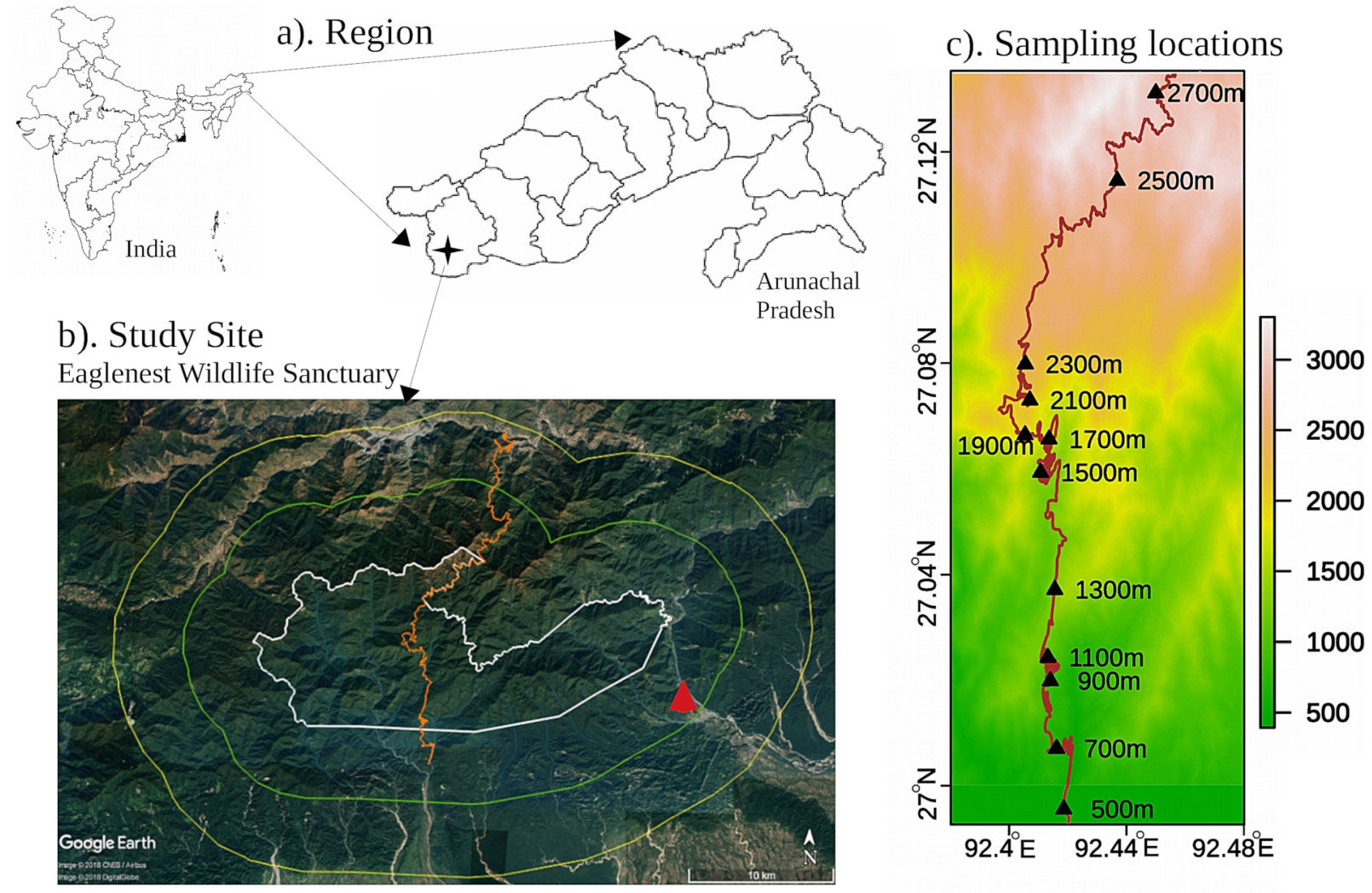

651 Figure 1. Study site in Eaglenest wildlife sanctuary, India. a). Location of the study site in

652 West Kameng district, Arunachal Pradesh, north-east India b). A google earth image of

653 Eaglenest Wildlife Sanctuary with the the boundary of the Protected Area (PA) marked in

654 white, and the $5 \mathrm{~km}$ buffer strip in green. The dirt track running through the sanctuary

655 traverses elevations from $100 \mathrm{~m}$ in the south to the pass at $2780 \mathrm{~m}$ and down to $1200 \mathrm{~m}$ to

656 the north. is shown in orange, and the $200 \mathrm{~m}$ sampling location, which was outside the PA

657 is marked by a red triangle. c). Digital elevation map showing the Eaglenest track and the

658 sampling locations between $500 \mathrm{~m}$ and $2700 \mathrm{~m}$. The $200 \mathrm{~m}$ sampling site is shown by a 659 red triangle in the Google image. 

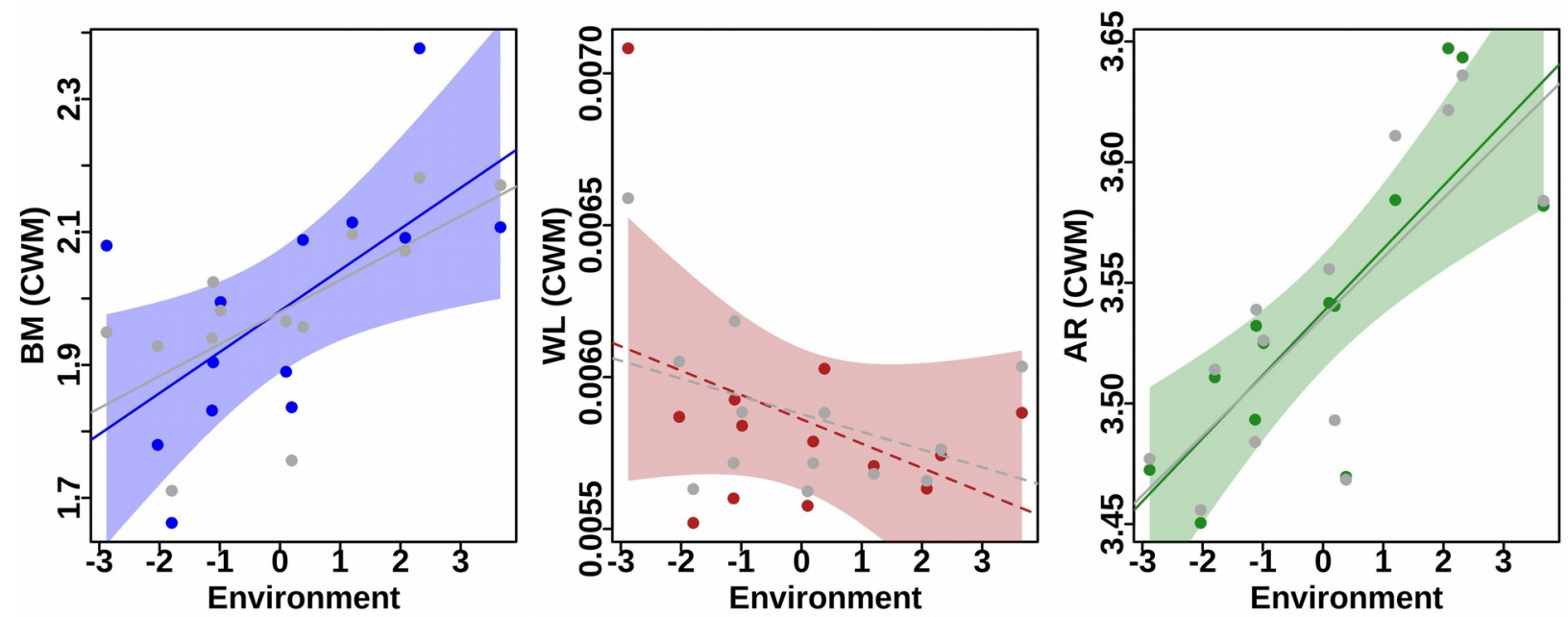

660 Figure 2. Variation of hawkmoth community traits along an Environment. The plots show

661 the change in community weighted means (CWM) of body mass (BM), wing loading (WL)

662 and wing aspect ratio (AR) plotted against composite Environment (which has a strong 663 positive correlation with elevation). For each trait, the colored representations are for

664 CWM1 (population mean trait values weighted by local abundance) while the grey are for

665 CWM2 (regional species mean trait values weighted by local abundance). The lines

666 represent the best fits, with the ones significant at 95\% shown in solid and the others with 667 dashes. 


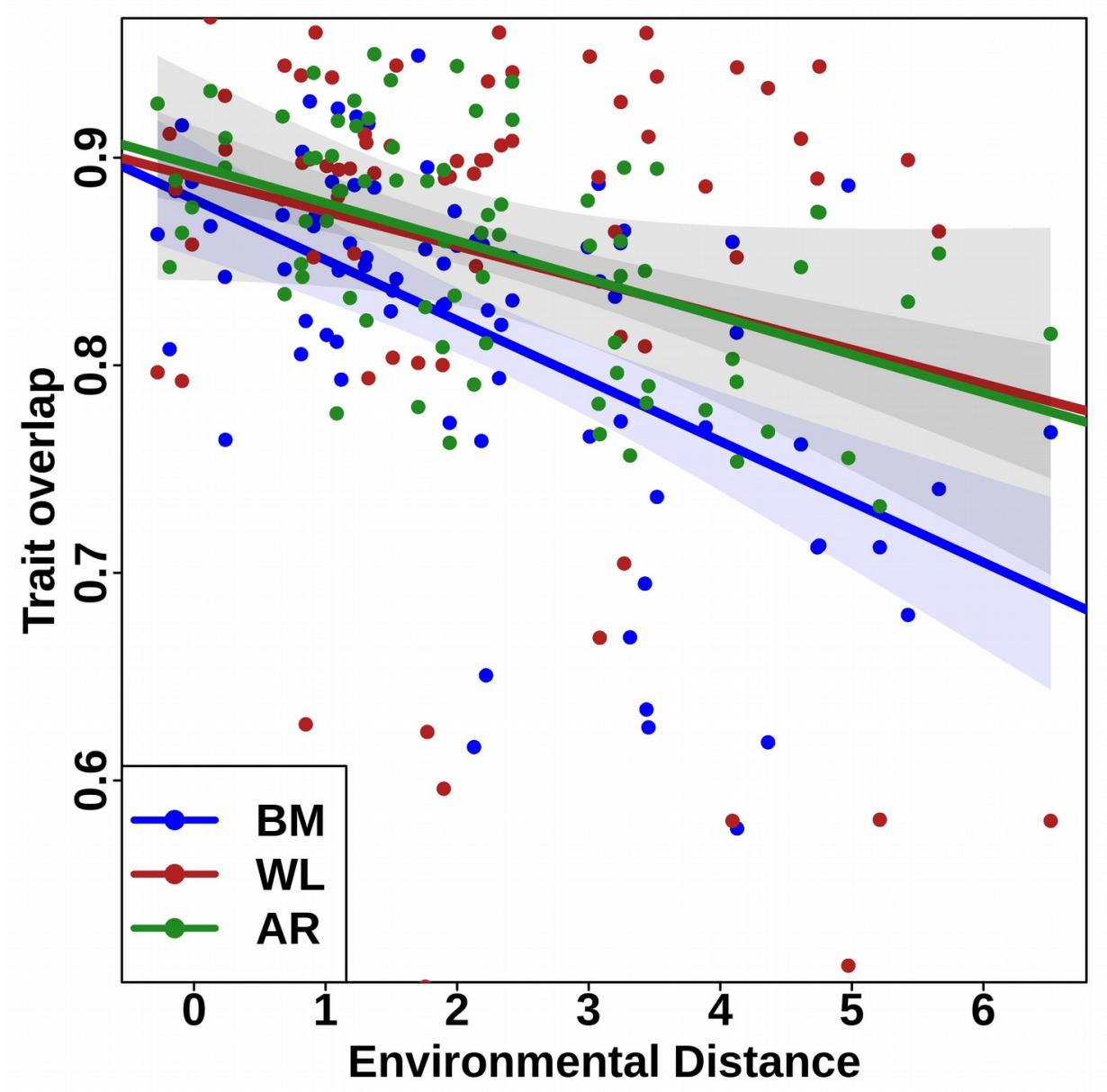

668 Figure 3. Relationship between trait overlap and environmental distance. The plot shows

669 the scatter and the regression line for the relationship between the overlap in trait

670 distribution functions for pairs of communities and the Environmental distance between

671 them. The shaded areas are the 95\% errors on the slope. The three traits analysed are

672 body mass (BM), wing loading ( $\mathrm{WL}$ ), and wing aspect ratio (AR). The statistically

673 significant correlation suggests the importance of these as key functional response traits. 

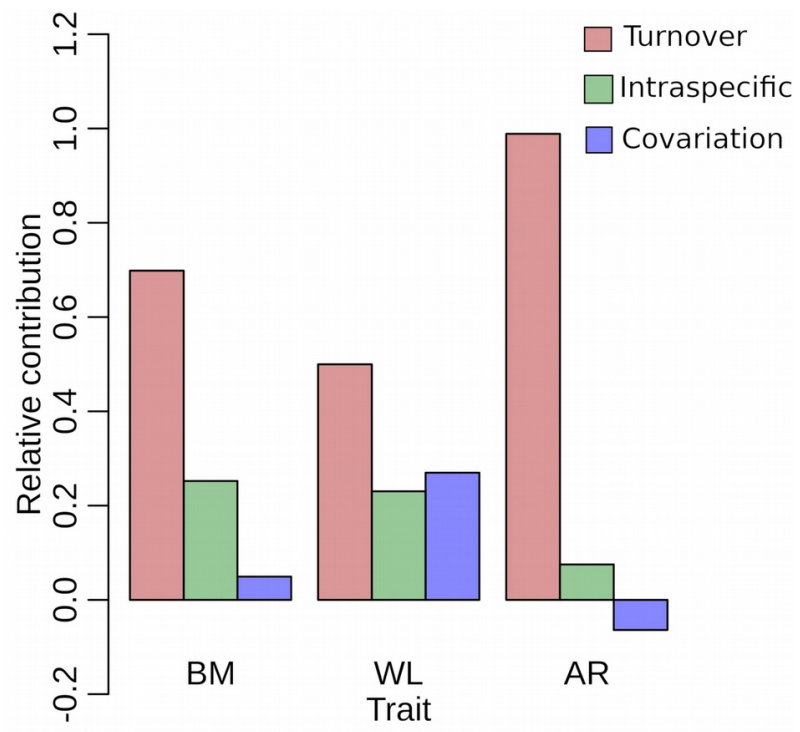

683

685 Figure 4. Decomposition of variation of hawkmoth community functional traits along an 686 Environmental gradient. The contributions of species turnover and intraspecific variation

687 (and their covariance) were calculated for body mass (BM), wing loading (WL) and wing 688 aspect ratio (AR) using the approach of Lepš et al., (2011). 

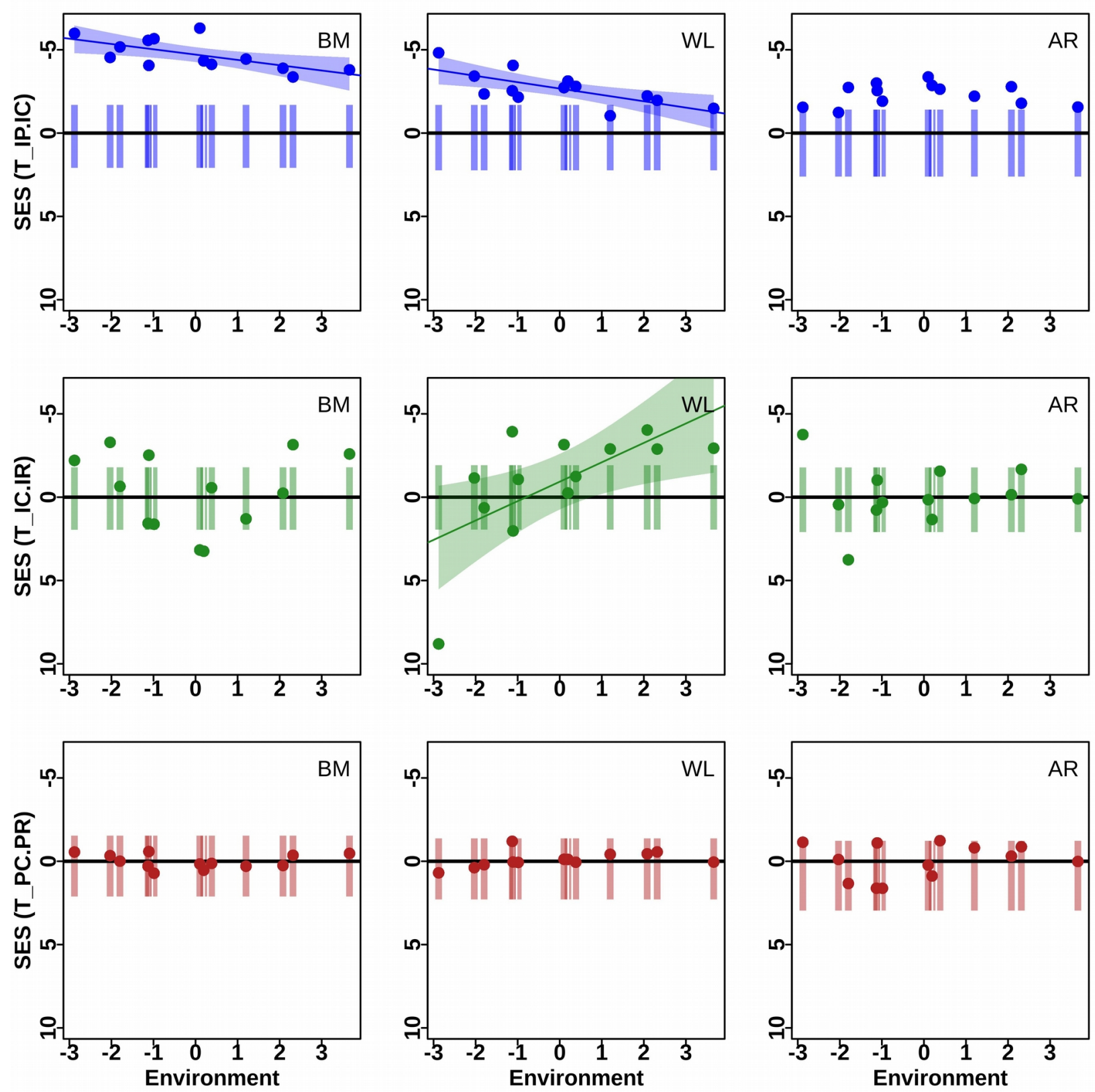

691 Figure 5. T-statistics of hawkmoth functional traits across an Environmental gradient. The

692 plots show the T-statistics for 3 traits: body mass (BM), wing loading (WL) and wing aspect 693 ratio $(A R)$, and for each of the 13 elevational communities (along the $x$-axis) in terms of the 694 standardized effect size (SES) along the y-axis. The vertical bars are the 95\% distribution 695 from 999 simulated null communities, and the dots are the observed community values.

696 The three T-statistics variance ratios are (a) $\mathrm{T}_{\mathrm{IP} / \mathrm{IC}}$ - within-population to within-community; 
697 (b) $T_{I C / R}$ - within-community to within-regional, assessed using individual values; and (c)

$698 \mathrm{~T}_{\mathrm{PC} / \mathrm{PR}}$ - within-community-wide to within-region, assessed using population-means.

699 Additionally, linear regression fits including errors (shaded region) have been shown for

700 metrics which are significantly correlated $(\mathrm{Cl}>95 \%)$ with the composite Environmental

701 variable (which is positively correlated with elevation). 


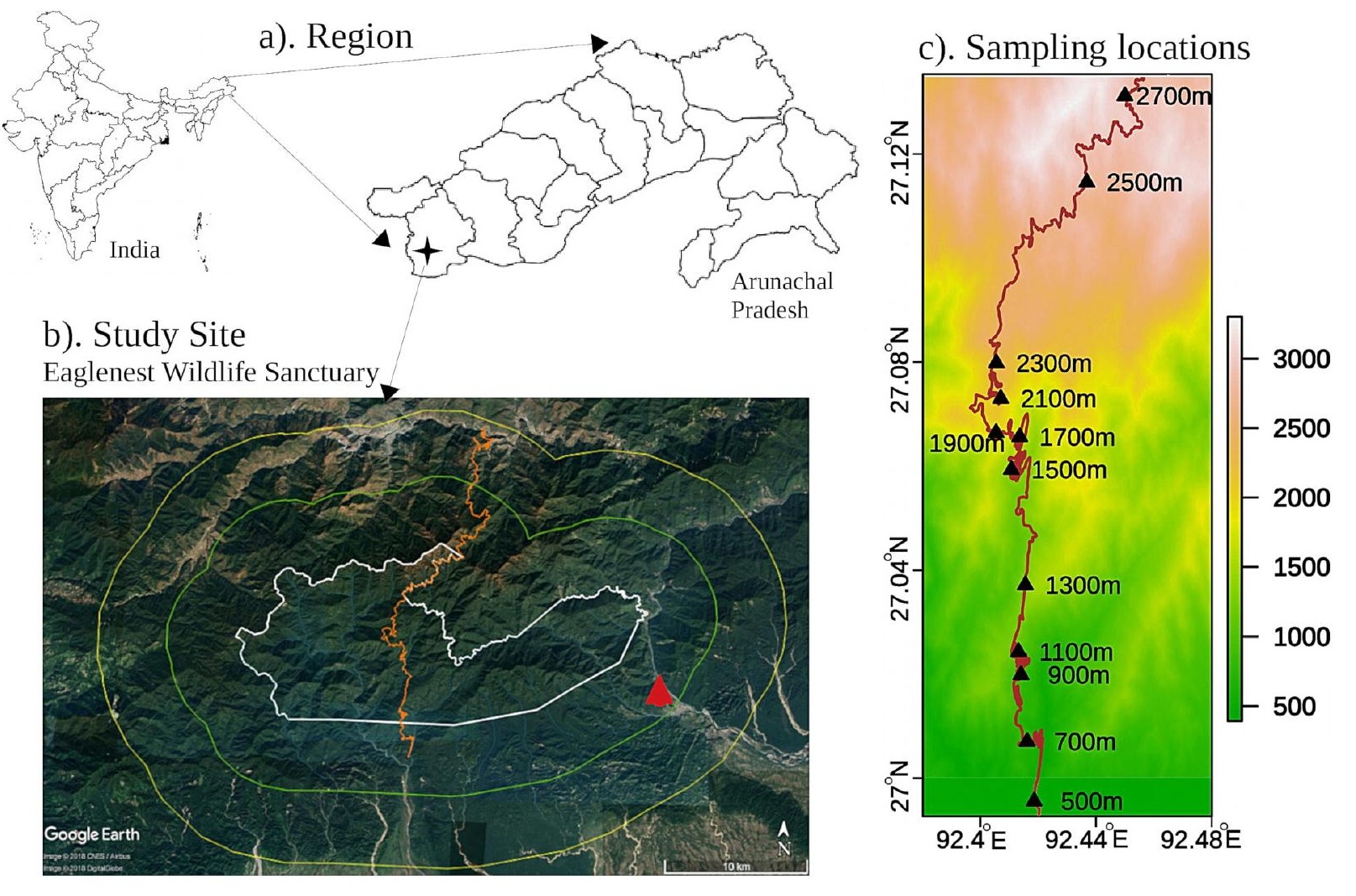




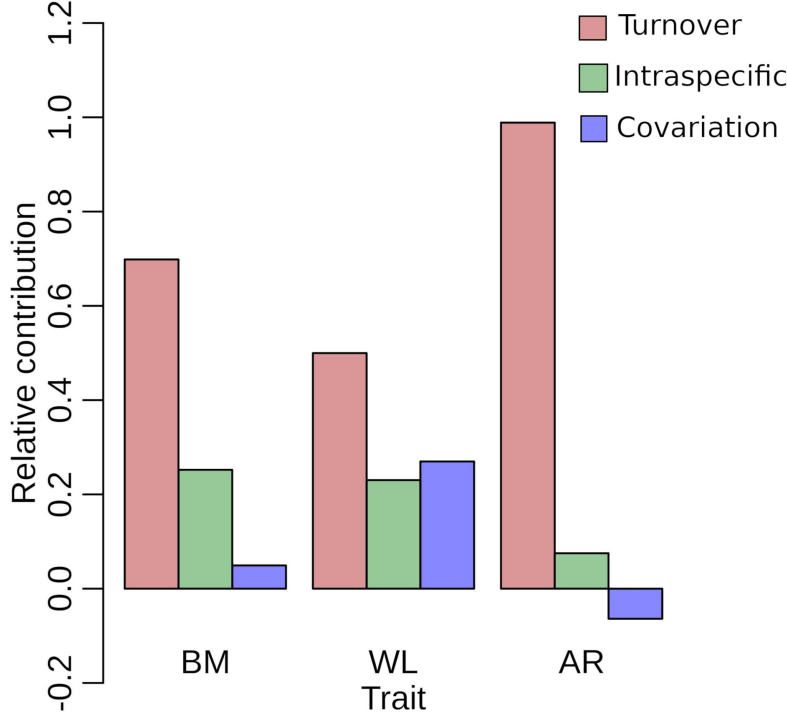


